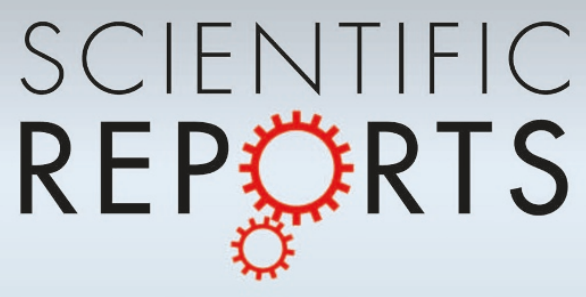

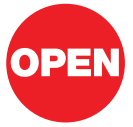

SUBJECT AREAS:

COLLOIDS

APPLIED PHYSICS

SYNTHESIS AND PROCESSING

NANOPARTICLES

Received

6 March 2013

Accepted

15 April 2013

Published

29 April 2013

Correspondence and requests for materials should be addressed to C.H.L. (chliang@issp. ac.cn)

\section{Spontaneous Growth and Chemical Reduction Ability of Ge Nanoparticles}

\author{
Jun Liu ${ }^{1,2}$, Changhao Liang ${ }^{1}$, Zhenfei Tian' ${ }^{1}$ Shuyuan Zhang ${ }^{2} \&$ Guosheng Shao ${ }^{3}$
}

'Key Laboratory of Materials Physics and Anhui Key Laboratory of Nanomaterials and Nanotechnology, Institute of Solid State Physics, Hefei Institutes of Physical Science, Chinese Academy of Sciences, Hefei, 23003 1, China, ${ }^{2}$ Department of Materials Science and Engineering, University of Science and Technology of China, Hefei, 230026, China, ${ }^{3}$ Institute for Renewable Energy and Environmental Technologies, University of Bolton, Bolton BL3 5AB, UK.

Forming colloidal solutions containing semiconductor quantum-sized nanoparticles (NPs) with clean surface has been a long-standing scientific challenge. In this contribution, we report a "top-down" method for the fabrication of Ge NPs by laser ablation of a Ge target in deionized water without adding any stabilizing reagents. The initial Ge NPs in amorphous structure showed spontaneous growth behavior by aging Ge colloids in deionized water under ambient temperature, which gradually evolved into a metastable tetragonal structure as an intermediate phase and then transformed into the stable cubic structure, being consistent with the Ostwald's rule of stages for the growth in a metastable system. The laser-induced initial Ge NPs demonstrate a unique and prominent size-dependent chemical reductive ability, which is evidenced by the rapid degradation of organic molecules such as chlorinated aromatic compounds, organic dyes, and reduction of heavy metal $\mathrm{Cr}(\mathrm{VI})$ ions.

$t$ has always been the focus of intense scientific efforts in nanomaterials and nanotechnology to explore unusual physical and chemical properties of colloidal nanoparticles (NPs), particularly those induced by quantum size effects and/or surface effects ${ }^{1-6}$. Group IV elemental semiconductors, such as Si and Ge NPs, have wide applications in optic and electronic devices, as well as in biological fluorescence imaging because of their size-dependent optical properties and relatively low toxicity ${ }^{7-11}$. Ge has a larger excitonic Bohr radius $(24.3 \mathrm{~nm})$ than $\mathrm{Si}(4.9 \mathrm{~nm})$, which consequently makes it more prominent in studies of quantum confinement effects ${ }^{12,13}$. Size-dependent photoluminescence of Ge NPs has been explored extensively by many research groups. Prabakar and colleagues $^{14}$ synthesized allylamine-terminated Ge nanocrystals with average sizes ranging from $4.0 \mathrm{~nm}$ to $5.5 \mathrm{~nm}$, which displayed size-dependent strong photoluminescence with wavelength between $420 \mathrm{~nm}$ and $480 \mathrm{~nm}$ and were used as optical probes for HepG2 cell imaging. Ge NPs can be modified with various functional groups, such as alcohols, esters, chloride, alkyl, and polymers, which are used in organometallic chemistry as carriers or catalysts ${ }^{15-18}$. Considerable efforts have been made in the past on embedding Ge NPs in a dielectric matrix, particularly amorphous $\mathrm{SiO}_{2}$, for their potential applications in optoelectronic, photovoltaic, and nonvolatile memory devices ${ }^{19-21}$.

Undoubtedly, freestanding Ge NPs without surfactant covered on the surfaces are more beneficial for exploring their physicochemical properties. Overall Ge NP surface is always functionalized with organic reagents to prevent oxidization and aggregation in conventional "bottom-up" solution chemical routes, which have been used to obtain stable and well-dispersed Ge NPs. Understandably, such functionalized surface impedes intrinsic properties of Ge NPs to some extent. Besides, Ge NPs with naked surfaces are critical for potential applications in the environmental and biomedical fields. Motivated by these considerations, the current study utilized the laser ablation in liquids (LAL) technique, which is a "top-down" method, to transfer materials from the bulk Ge target into the liquid as highly dispersed Ge NPs, without using any stable reagents. This technique was recently used for the fabrication of NPs of a wide range of elemental and compound materials ${ }^{22-29}$. Here we report that the resultant Ge NP containing hydrosol is a metastable thermodynamic system. The NPs in such a colloidal system were observed to experience spontaneous growth during aging at the ambient temperature in a sealed chamber, when gradual particle growth was accompanied with changes in phase structures. The growth of elemental Ge evidently coincides with Ostwald's rule of stages, as empirically described by Ostwald in 1897 and dubbed the rule of stages $^{30}$. In Ostwald's picture of crystallization, intermediate phases may exist during the transition from an amorphous to a final thermodynamically stable structure. We also report unusual optical absorption characteristics and unique chemically reductive ability of LAL-induced Ge NP colloids by rapid decomposition of organic pollutants, including chlorinated aromatic compounds, organic dyes, and reduction of heavy metal ions. In 
contrast, these intrinsic physicochemical properties of Ge have rarely been achieved in normal "bottom-up" chemical route-synthesized Ge NPs.

\section{Results}

Fig. 1a presents a low-magnification transmission electron microscopy (TEM) image of Ge NPs collected from fresh colloidal solutions. A large amount of small quasi-spherical NPs was observed. The size distribution of Ge NPs was derived by calculation and fitting of about $400 \mathrm{NPs}$ from more TEM images. Average diameter of fresh Ge NPs ranges from $7.0 \mathrm{~nm}$ to $8.0 \mathrm{~nm}$ according to the size distribution histogram as shown in Fig. 1c. The high-resolution TEM image (HRTEM, Fig. 1b) and its corresponding select area electronic diffraction (SAED) pattern (inset at the upper right corner) indicate that fresh Ge NPs are almost in an amorphous state, which is also demonstrated in the X-ray powder diffraction (XRD) pattern in Fig. $1 \mathrm{j}$ of fresh Ge NPs.

We found that the initially amorphous Ge NPs are in a metastable state. The morphology and structure of Ge NPs would be changed to reduce surface energy and to attain a more stable structure by simply aging fresh Ge colloidal hydrosol in a sealed chamber at the ambient temperature. With the aging time of 3 hours, we can observe that the Ge NPs self-assembled into fractal aggregates with size approximate to $100 \mathrm{~nm}$ (as shown in Fig. 1d). The corresponding XRD pattern (in Fig. 1j) from the water-containing samples indicated the amorphous Ge NPs grows into Ge NPs in tetragonal and/or cubic phase structure during the aging process. The tetragonal phase were reported as a pressure induced phase in a number laboratories using a variety of techniques ${ }^{31-34}$. However, there have been no reports on phase transitions detected in a nanosized Ge colloidal system under ambient atmosphere. The diffraction peaks located at $27.2^{\circ}, 45.3^{\circ}$, and $53.7^{\circ}$ can be accurately indexed to the cubic phase Ge (JCPDS Card No. 00-004-0545, Fd-3m (227) ), which is a typical diamond structure $(\mathrm{Ge}-\mathrm{I})^{31}$ and the stable state of $\mathrm{Ge}$ at room temperature and normal pressure. The diffraction peak at $30.9^{\circ}$ belongs to the tetragonal Ge phase in a body-centered tetragonal structure (Ge-III at $10.8 \mathrm{GPa})^{34}$, a metastable phase. The broad background peak located around $22^{\circ}$ in two theta degree is resulted from the amorphous glasssubstrate for holding the samples during the XRD measurements. The SAED pattern also displayed diffraction rings belong to two different structures (as shown in Fig. 1e), we can observe the diffraction rings (marked with blue curves) matched with (111) and (220) crystallographic planes of Ge-I, respectively. And other diffraction rings can be well assigned to the (012) crystallographic planes of GeIII (at $10.8 \mathrm{GPa}$ ), and (211), (222), (004) crystallographic planes of Ge-III (at $1 \mathrm{~atm})^{34}$. The HRTEM image (Fig. 1f) taken from the selected area in Fig. 1d clearly exhibits the (012) and (211) planes with interplanar spacing of $2.88 \AA$ and $2.48 \AA$ for Ge-III NPs, respectively. More evidence from TEM analysis is shown in Supplementary Fig. S2-S3 online. The phase transition of Ge NPs carried on by prolonging the aging time. Fig. $1 g$ presents typical large Ge spheres with an average size of $350 \mathrm{~nm}$ owing to self-assembling from the initial small Ge NPs after 24 hours of aging and the average size was estimated using about 60 spheres in more TEM images. Fig. $1 \mathrm{~h}$ is the corresponding SAED pattern of a single Ge sphere, indicating the polycrystalline nature. The HRTEM image (Fig. 1i) of the selected area in Fig. 1g evidently displays a number of grain boundaries and lattice distortions, which reflect that spontaneous self-assembling
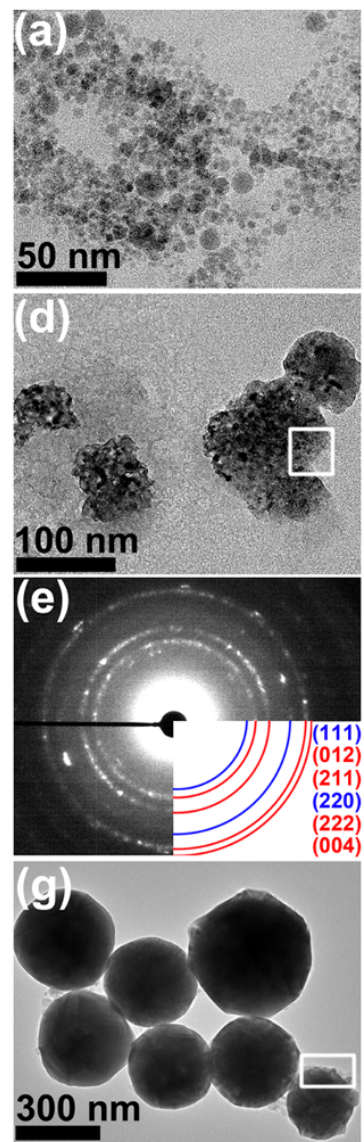
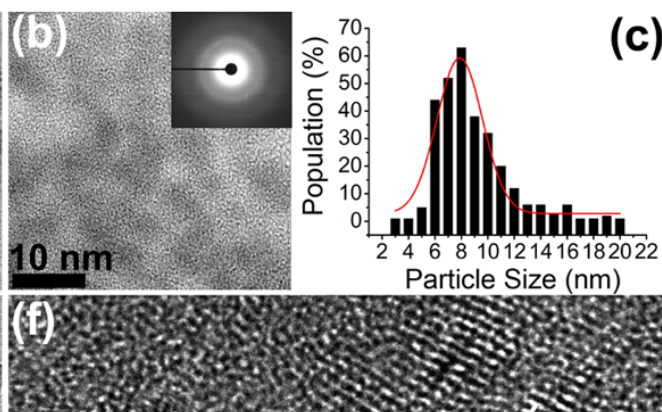

(c) (j)
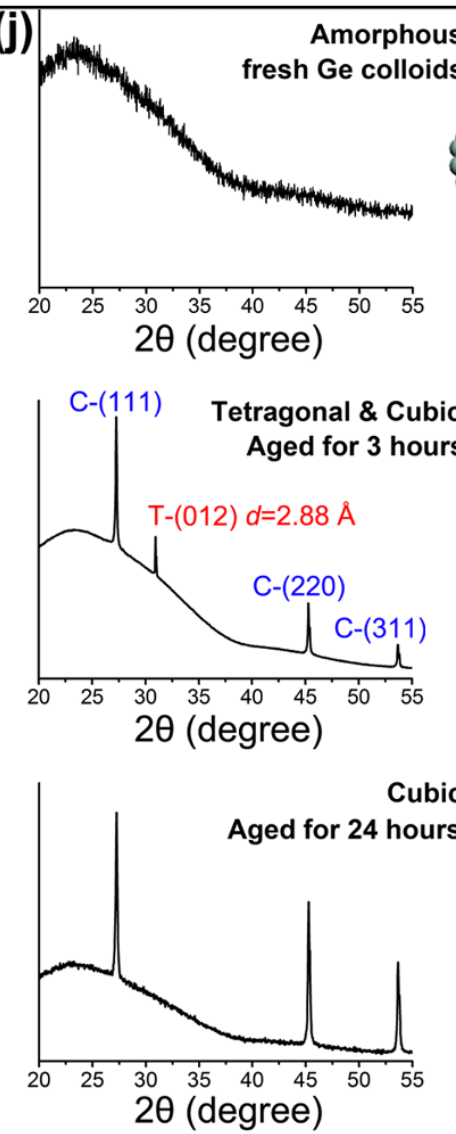
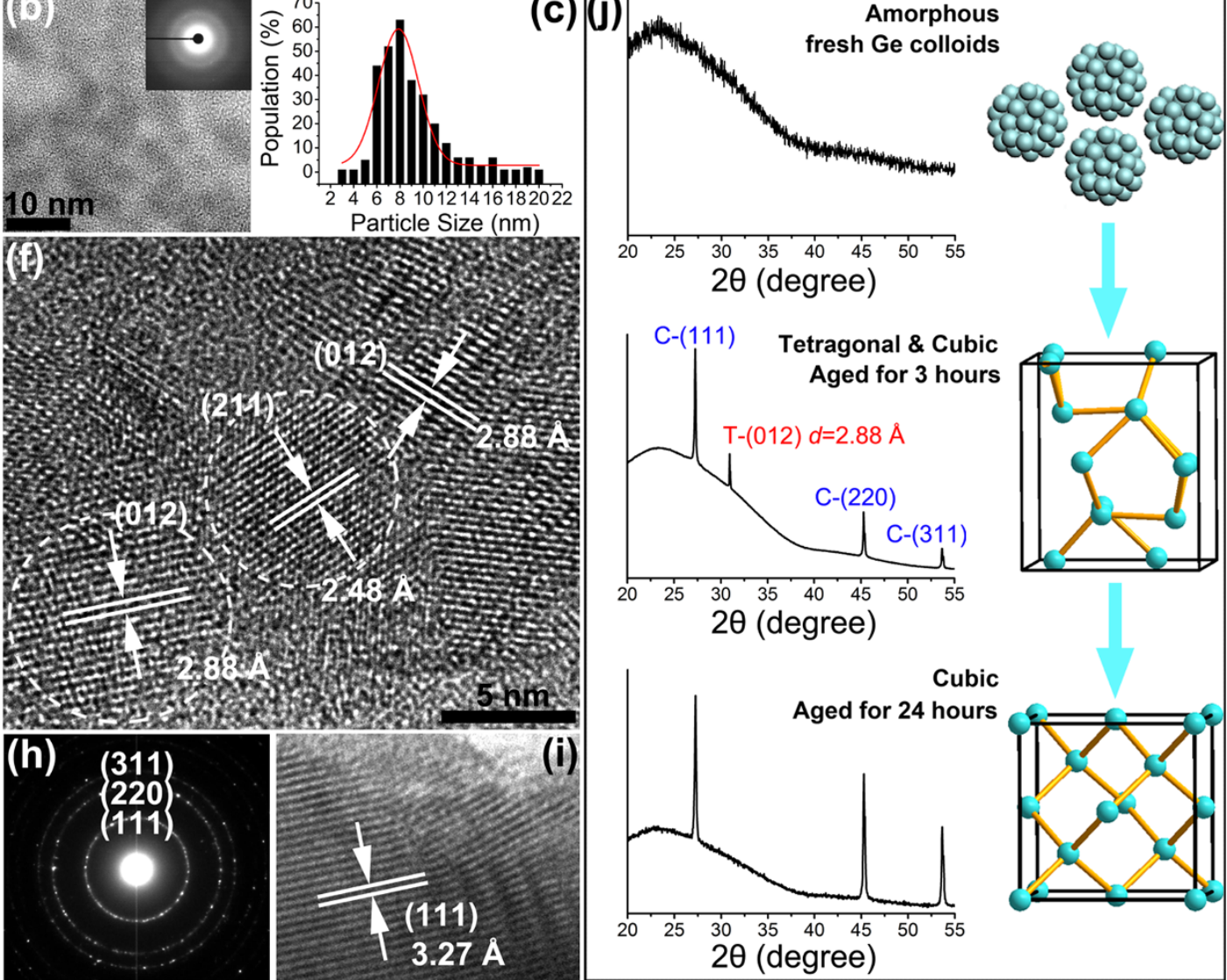

Figure $1 \mid$ (a-b) TEM images of fresh Ge NPs; with inset in (b) the corresponding SAED pattern. (c) Size histogram of fresh Ge NPs. (d-f) Low-magnification TEM image, SAED pattern, and HRTEM image of selected area in (d) for Ge NPs with aging time of 3 h. (g-i) Low-magnification TEM image, SAED pattern, and HRTEM image of selected area in (g) for Ge NPs with aging time of 24 h. (j) XRD patterns of Ge NPs with different aging time. 

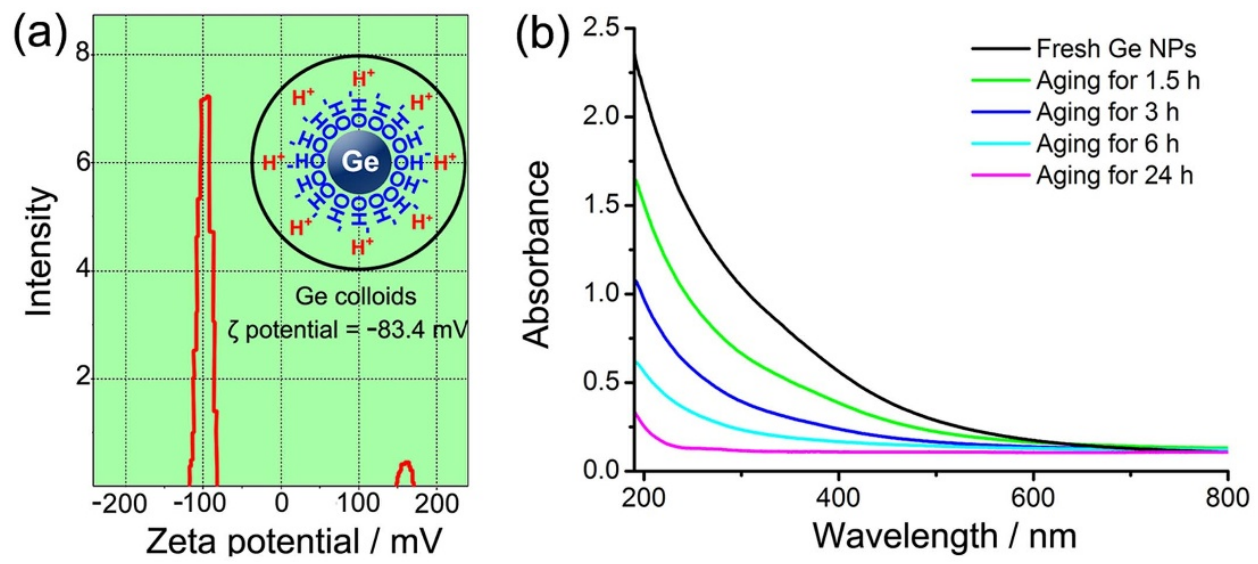

Figure $2 \mid$ (a) Zeta potential of fresh Ge NPs suspended in deionized water. Inset of b is the schematic configuration of charged fresh Ge NPs. (b) UV-vis spectra of Ge NPs with aging time from 0-24 hours.

and crystallization of fresh amorphous Ge NPs into large crystalline spheres definitely occurred under ambient temperature. The XRD pattern of assembled Ge spheres in Fig. $1 \mathrm{j}$ showed that all peaks can be indexed to the stable Ge-I structure and the peak located at $30.9^{\circ}$ that belongs to the Ge-III structures disappeared. This indicates that the transition of Ge-III $\rightarrow$ Ge-I occurred in deionized water during the aging process. It is necessary to point out that our experiments showed that the metastable Ge-III structures can be maintained if the Ge NPs are kept as dry powders, wherein the Ge-III will not convert into Ge-I at the ambient temperature.

The Raman spectra for Ge NPs with aging time of $3 \mathrm{~h}$ and $24 \mathrm{~h}$ are presented in Supplementary Fig. S4 online. According to the results reported by Kobliska ${ }^{35}$, the Raman peak at $274 \mathrm{~cm}^{-1}$ is attributed to the Ge-III structures and the peaks at $297 \mathrm{~cm}^{-1}$ and $299 \mathrm{~cm}^{-1}$ should be assigned to the Ge-I structures. The change in the Raman spectra of these two Ge NPs samples further demonstrate that the presence of Ge-III and the transition of Ge-III $\rightarrow$ Ge-I occurred by aging amorphous Ge NPs in a liquid phase under ambient temperature. Spontaneous growth of initial amorphous Ge NPs during aging the colloidal system is consistent with energetic minimization. The structural evolution in the metastable colloidal system agrees well with the classical Ostwald's rule of stages, which were mostly observed in colloids and proteins. Ostwald's rule of stages for quantum dot growth in inorganic crystals such as metal chalcogenide and $\mathrm{LiFePO}_{4}{ }^{36,37}$, have also been reported, however, rare evidence is available in the growth of elemental NPs. Such an evolution of growth stages indicates that the energy of formation $\mathrm{E}_{\mathrm{f}}$, for the observed phases is in the sequence of $\mathrm{E}_{\mathrm{f}}(\mathrm{am})>\mathrm{E}_{\mathrm{f}}(\mathrm{Ge}-\mathrm{III})>\mathrm{E}_{\mathrm{f}}$ (Ge-I). This is not shocking, as the amorphous structure, which is usually dubbed as a frozen liquid, is probably the least stable with respect to the two crystalline phases of which the diamond structure is the ground-state phase. Further theoretical modeling for structural evolution in the colloidal system is desirable for enhanced understanding of the stabilizing process.

Our experiments showed that LAL-derived Ge NPs were welldispersed in deionized water without using any stable reagents as surfactants in the initial state. This may be attributed to charged surfaces and drastic Brownian motions of small fresh Ge NPs. As shown in the inset of Fig. 2a, fresh LAL-derived Ge NPs are surrounded by an elastic hydration layer and its corresponding zeta potential pattern displays a high $\zeta$ potential of $-83.4 \mathrm{mV}$ measured by MALVERN instrument (Zetasizer3000HSa). Generally, the colloidal solutions are considered to be stable if the zeta potential is above $30 \mathrm{mV}$ for positively charged NPs or below $-30 \mathrm{mV}$ for negatively charged $\mathrm{NPs}^{38}$. Therefore, the measured zeta potential of Ge NPs is more negative than the critical value $(-30 \mathrm{mV})$, which can effectively enhance the surface repulsion force of Ge NPs to prevent aggregation and sedimentation. Fig. 2b shows UV-vis spectra (ranging from $190 \mathrm{~nm}$ to $800 \mathrm{~nm}$ ) of Ge NPs suspended in deionized water with different aging time under ambient temperature in darkness. The absorbance increases gradually with decreasing wavelength ${ }^{39,40}$, however, there is no characteristic absorption band edges corresponding to that for bulk $\mathrm{Ge}^{29,41,42}$ and those for other solution chemistry-synthesized Ge NPs reported previously ${ }^{43-45}$. As we know, the optical absorption properties measured under the transmission mode is effective when the particle sizes in the suspension are below the size for complete optical absorption. With the increase of aging time, the fractal or globular aggregates formed by the initial small Ge NPs experienced growth and the amount of adequately small Ge NPs decreased in the solution samples, so that the intensity of optical absorptions declined gradually ${ }^{46}$. However, the optical absorption edge did not change, even though phase structure and crystallinity both changed during aging.

\section{Discussion}

In order to investigate the functionalities of the Ge NPs, we attempted to use LAL-induced Ge NPs for the chemical decomposition and reduction of various organic molecules and metal ions, such as 2,5-dichlorophenol, methyl orange (MO), and $\mathrm{Cr}$ (VI) ions, to test size effects of Ge NPs on chemical reductive ability. An aqueous solution with $16 \mathrm{mg} / \mathrm{L}$ 2,5-dichlorophenol was alkaline with a $\mathrm{pH}$ value of 11.0, which was used to react with fresh amorphous Ge NPs. The standard electrode potential of Ge was $-1.03 \mathrm{~V}$, thus, electrode potential was $-1.25 \mathrm{~V}$ in $16 \mathrm{mg} / \mathrm{L} 2,5$-dichlorophenol aqueous solutions, as calculated from the Nernst equation (see Supplementary Equation S1 online). This value is much larger than the standard electrode potential of zero-valent iron $(-0.44 \mathrm{~V})$, which is widely used as a dechlorinating agent ${ }^{47-51}$.

In this heterogeneous reaction system, first of all, the 2,5-dichlorophenol molecular will absorb on the surface of fresh Ge NPs. Then

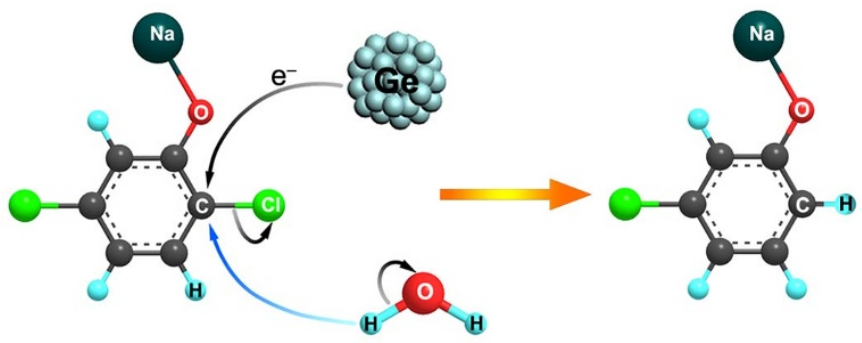

Figure 3 | Dechlorination mechanisms for fresh amorphous Ge NPs react with $16 \mathrm{mg} / \mathrm{L}$ 2,5-dichlorophenol $(\mathrm{pH}=11.0)$. 

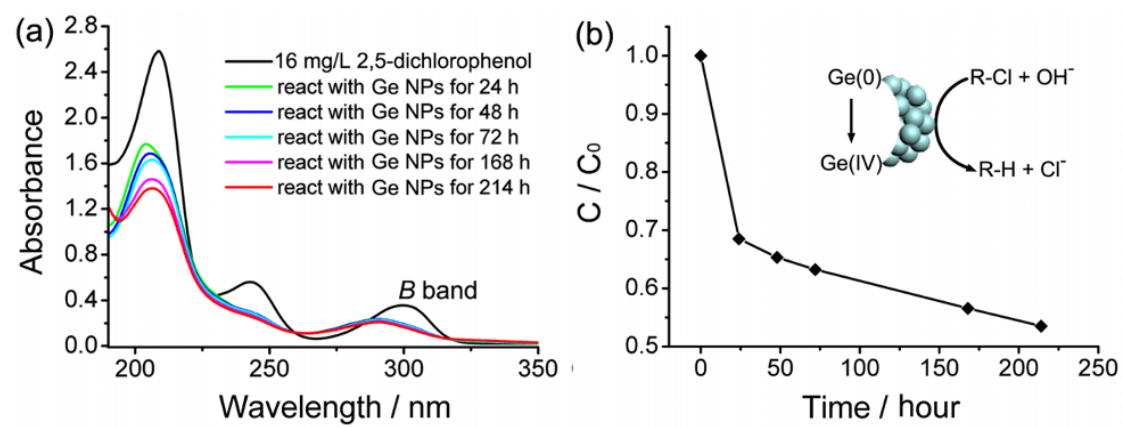

Figure $4 \mid$ (a) UV-vis absorbance spectra of 2,5-dichlorophenol after interacting with fresh Ge NPs at different durations; (b) plotted dechlorination rate of (a).

the $\mathrm{Ge}$ atoms will donate their valence electrons to the $\mathrm{C}$ atoms and break up the corresponding $\mathrm{C}-\mathrm{Cl}$ bond, as is schematically shown in Fig. 3. The work function of the valence electrons of Ge atoms depend on the size of Ge NPs, and in the nano-scale the work function is expected to decrease with reducing particle size through the associated reduction in the binding energy. The lowered work function in the donor type catalyst helps enhance the potential difference across the reacting interface, thus promoting charge transfer to facilitate the following overall chemical reaction for reduction:

$$
\mathrm{Ge}(0)+\mathrm{R}-\mathrm{Cl}+\mathrm{OH}^{-} \rightarrow \mathrm{Ge}(\mathrm{IV})+\mathrm{R}-\mathrm{H}+\mathrm{Cl}^{-}
$$

where $\mathrm{R}$ - represents the $-\mathrm{C}_{6} \mathrm{H}_{3} \mathrm{ClO}^{-} \mathrm{Na}^{+}$group.

Fig. 4a shows UV-vis absorbance spectra of 2,5-dichlorophenol after reacting with fresh Ge NPs. It exhibits that as dechlorination proceeded, $\mathrm{Cl}$ atoms, as typical auxochromes, broke away from benzene rings, leading to a decline in absorbance of the three representative absorption bands of 2,5-chlorinated sodium phenolate, together with an obvious blue shift, especially for $B$ band or finestructure band located at $300 \mathrm{~nm}$ of benzene $\mathrm{e}^{52}$. As shown in Fig. $4 \mathrm{~b}$, the sharp decrease in reaction rate could be attributed to the low work function of fresh Ge NPs. The concentration of 2,5-dichlorophenol rapidly decreased to $68 \%$ in 24 hours; however, dechlorination rate slowed down subsequently. Only about $15 \%$ of 2,5 -dichlorophenol was reduced in $192 \mathrm{~h}$. But the large assembled Ge nanospheres revealed no evident reaction with 2,5-dichlorophenol. These results do indicate obvious size-dependent reductive ability of Ge NPs. Only fresh Ge NPs exhibit effective dechlorinating capability similar to that of zero-valent iron NPs.

Chemical reductive ability of fresh Ge NPs was also demonstrated for the removal of $25 \mathrm{mg} / \mathrm{L} \mathrm{MO}$ aqueous solution, which is a widely used azo dye. The fresh Ge NPs, acting as reductive reagent, donated electrons to break the $\pi$-bond in the azo double bond. The corresponding removal process is shown in Supplementary Scheme S1 online. However, the N-N $\sigma$ bond or the benzene rings are difficult to destroy by the same redox process. Therefore, we can clearly observe that three representative absorption bands of benzene, namely, the $E_{1}, E_{2}$, and $B$ bands were obviously blue shifted because of the disappearance of chromophore molecular structures (see Supplementary Fig. S5a online). In addition, the fresh Ge NPs can effectively remove heavy metal ions, such as $\mathrm{Cr}$ (VI), which exists widely in polluted water sources and soil. Supplementary Equation $\mathrm{S} 2$ online shows that a $12 \mathrm{mg} / \mathrm{L} \mathrm{K}_{2} \mathrm{Cr}_{2} \mathrm{O}_{7}$ aqueous solution, as a source of $\mathrm{Cr}(\mathrm{VI})$, reacted with fresh Ge NPs. The corresponding UV-vis spectra (see Supplementary Fig. S5b online) indicate that the concentration of $\mathrm{Cr}(\mathrm{VI})$ ions decreased quickly to half in $10 \mathrm{~min}$. The $\mathrm{Cr}(\mathrm{VI})$ ions almost disappeared after $90 \mathrm{~min}$. The inset in Supplementary Fig. S5b online shows that the reacted solution became almost colorless, when $\mathrm{Cr}$ existed mainly as $\mathrm{Cr}$ (III) in the solution $^{53,54}$

In summary, the LAL technique, which is a "top-down" method, can be used to produce a well-dispersed colloidal semiconductor of
Ge NPs without using any protective organic agents. The quantumsize and clean surface of LAL-induced Ge NPs are beneficial for exploring intrinsic physicochemical properties of semiconductor Ge NPs. For the first time, we report an interesting spontaneous growth process of the metastable Ge NPs and associated structural evolution from an initial amorphous state to a final cubic structure, with a tetragonal structure as an intermediate phase. The LALderived Ge NPs significantly exhibit excellent chemical reductive ability similar to that of zero-valent iron NPs. Various organic molecules, such as 2,5-dichlorophenol, MO, can be quickly degraded and metal $\mathrm{Cr}(\mathrm{VI})$ ions can be reduced, particularly by using fresh and small Ge NPs. The LAL-induced Ge NPs with clean surface may find special applications in the biomedical field, as well as in catalysis and in battery, while due to the instability of the colloidal system it may be necessary to introduce further surface functionalization with additional molecules or by being composited with other structures.

\section{Methods}

“Top-down" synthesis of quantum-sized Ge NPs. A piece of crystallographic Ge target (cubic, 99.99\%, as shown in Supplementary Fig. S1 online) was immersed in $15 \mathrm{~mL}$ deionized water in a cell, which was ablated for designated durations using a $1064 \mathrm{~nm}$ Nd:YAG pulse laser with a pulse duration of $10 \mathrm{~ns}$ and pulse energy of $100 \mathrm{~mJ}$. Well-dispersed fresh colloidal Ge NPs were obtained in water by ablating for 5 min. Aging treatment of fresh Ge colloids was conducted in a sealed chamber, in a dark environment, at room temperature for different durations to monitor structure evolution of initial Ge NPs.

Structure characterization. X-ray powder diffraction (XRD) phase analysis of all collected products which are placed on a piece of glass slide substrate, was performed using a Philips X'Pert system with $\mathrm{Cu} \mathrm{K} \alpha$ radiation $(\lambda=1.5419 \AA$, scanning rate $=$ $1.0 \% \mathrm{~min}$ ). Field-emission scanning electron microscopy (SEM, Sirion 200 FEG) was used to trace size and morphology evolution of Ge NPs. Transmission electron microscopy (TEM, JEM-ARM 200F) was used to analyze the structure evolution of fresh Ge NPs. Shimadzu UV3600-MPC3100 spectrophotometer with a wavelength range from $190 \mathrm{~nm}$ to $800 \mathrm{~nm}$ was used to measure absorption spectra of products. MALVERN instrument (Zetasizer3000HSa) was used to measure zeta $(\zeta)$ potential of Ge NP colloids.

Chemical reductive treatment of various pollutants. Fresh Ge colloidal solution samples were obtained by ablating Ge target immersed in deionized water for certain minutes. As a reductive reagent, $15 \mathrm{ml} \mathrm{Ge}$ colloidal solution (density of Ge nanoparticles is $0.25 \mathrm{~g} / \mathrm{L}$ ) were added into $15 \mathrm{~mL}$ of $16 \mathrm{mg} / \mathrm{L} \mathrm{2,5-dichlorophenol} \mathrm{at}$ $\mathrm{pH}$ 11.0. The mixture was stirred and allowed to react at room temperature in a dark environment. Ultraviolet-visible (UV-vis) optical absorption spectra of reaction products were measured at certain temporal intervals after centrifugation to separate residual Ge NPs to monitor reactions between Ge NPs and pollutant molecules and/ or ions. In a similar way, the test for the removal of methyl orange was conducted by using $10 \mathrm{~mL} 25 \mathrm{mg} / \mathrm{L}$ methyl orange (MO; $\mathrm{pH}=7.0)$ and $20 \mathrm{~mL} 12 \mathrm{mg} / \mathrm{L} \mathrm{K}_{2} \mathrm{Cr}_{2} \mathrm{O}_{7}$ aqueous solution at $\mathrm{pH}$ 6.5. The amount of fresh Ge colloidal solution is $15 \mathrm{~mL}$ and $20 \mathrm{~mL}$, respectively.

1. Henglein, A. Small-particle research: physicochemical properties of extremely small colloidal metal and semiconductor particles. Chem. Rev. 89, 1861-1873 (1989).

2. Volokitin, Y. et al. Quantum-size effects in the thermodynamic properties of metallic nanoparticles. Nature, 384, 621-623 (1996).

3. Hagfeldtt, A. \& Grätzel, M. Light-induced redox reactions in nanocrystalline systems. Chem. Rev. 95, 49-68 (1995). 
4. Paulus, P. M. et al. Surface and quantum-size effects in Pt and Au nanoparticles probed by ${ }^{197} \mathrm{Au}$ Mössbauer spectroscopy. Phys. Rev. B 64, 205418 (2001).

5. Henglein, A. Physicochemical properties of small metal particles in solution: "Microelectrode" reactions, chemisorption, composite metal particles, and the atom-to-metal transition. J. Phys. Chem. 97, 5457-5471 (1993).

6. Stroyuk, A. L., Kryukov, A. I., Kuchmii, S. Y. \& Pokhodenko, V. D. Quantum size effects in the photonics of semiconductor nanoparticles. Theor. Exp. Chem. 41, 67-91 (2005).

7. Riabinina, D., Durand, C., Chaker, M., Rowell, N. \& Rosei, F. A novel approach to the synthesis of photoluminescent germanium nanoparticles by reactive laser ablation. Nanotechnology 17, 2152-2155 (2006).

8. Armatas, G. S. \& Kanatzidis, M. G. Size dependence in hexagonal mesoporous germanium: pore wall thickness versus energy gap and photoluminescence. Nano Lett. 10, 3330-3336 (2010).

9. Henderson, E. J., Hessel, C. M. \& Veinot, J. G. C. Synthesis and photoluminescent properties of size-controlled germanium nanocrystals from phenyl trichlorogermane-derived polymers. J. Am. Chem. Soc. 130, 3624-3632 (2008).

10. Brus, L. Luminescence of silicon materials: chains, sheets, nanocrystals, nanowires, microcrystals, and porous silicon. J. Phys. Chem. 98, 3515-3581 (1994).

11. Manhat, B. A. et al. One-step melt synthesis of water-soluble, photoluminescent, surface-oxidized silicon nanoparticles for cellular imaging applications. Chem. Mater. 23, 2407-2418 (2011).

12. Myung, N., Lu, X. M., Johnston, K. P. \& Bard, A. J. Electrogenerated chemiluminescence of Ge nanocrystals. Nano Lett. 4, 1183-1185 (2004).

13. Gu, G. et al. Growth and electrical transport of germanium nanowires. J. Appl. Phys. 90, 5747-5751 (2001).

14. Prabakar, S. et al. Size controlled synthesis of germanium nanocrystals by hydride reducing agents and their biological applications. Chem. Mater. 22, 482-486 (2010).

15. Tanke, R. S. et al. Synthesis of germanium nanoclusters with irreversibly attached functional groups: acetals, alcohols, esters, and polymers. Chem. Mater. 15 1682-1689 (2003).

16. Buriak, J. M. Organometallic chemistry on silicon and germanium surfaces. Chem. Rev. 102, 1271-1308 (2002).

17. Hanrath, T. \& Korgel, B. A. Chemical surface passivation of Ge nanowires. J. Am. Chem. Soc. 126, 15466-15472 (2004).

18. Knapp, D., Brunschwig, B. S. \& Lewis, N. S. Chemical, electronic, and electrical properties of alkylated Ge(111) surfaces. J. Phys. Chem. C 114, 12300-12307 (2010).

19. Stavarache, I., Lepadatu, A., Maraloiu, A. V., Teodorescu, V. S. \& Ciurea, M. L. Structure and electrical transport in films of Ge nanoparticles embedded in $\mathrm{SiO}_{2}$ matrix. J. Nanopart. Res. 14, 930 (2012).

20. Park, K. T. et al. Freestanding $\mathrm{Ge} / \mathrm{SiO}_{2}$ core/shell nanoparticles formed via metastable $\mathrm{SiO}_{2}$ hollow nanospheres on a Si wafer. Chem. Vap. Deposition 14, 331-333 (2008).

21. Yuan, C. L., Cai, H., Lee, P. S., Guo, J. \& He, J. Tuning photoluminescence of $\mathrm{Ge} / \mathrm{GeO}_{2}$ core/shell nanoparticles by strain. J. Phys. Chem. C 113, 19863-19866 (2009).

22. Liang, C. H., Shimizu, Y., Sasaki, T. \& Koshizaki, N. Synthesis of ultrafine $\mathrm{SnO}_{2-x}$ nanocrystals by pulsed laser-induced reactive quenching in liquid medium. J. Phys. Chem. B 107, 9220-9225 (2003).

23. Zhang, H. M., Liang, C. H., Tian, Z. F., Wang, G. Z. \& Cai, W. P. Single phase $\mathrm{Mn}_{3} \mathrm{O}_{4}$ nanoparticles obtained by pulsed laser ablation in liquid and their application in rapid removal of trace pentachlorophenol. J. Phys. Chem. C 114, 12524-12528 (2010).

24. Liu, J., Liang, C. H., Zhang, H. M., Zhang, S. Y. \& Tian, Z. F. Silicon-doped hematite nanosheets with superlattice structure. Chem. Commun. 47, 8040-8042 (2011).

25. Liang, C. H., Shimizu, Y., Masuda, M., Sasaki, T. \& Koshizaki, N. Preparation of layered zinc hydroxide/surfactant nanocomposite by pulsed-laser ablation in a liquid medium. Chem. Mater. 16, 963-965 (2004).

26. Zhang, H. M. et al. Defect-mediated formation of Ag cluster-doped $\mathrm{TiO}_{2}$ nanoparticles for efficient photodegradation of pentachlorophenol. Langmuir 28, 3938-3944 (2012).

27. Yang, G. W. Laser ablation in liquids: applications in the synthesis of nanocrystals. Prog. Mater. Sci. 52, 648-698 (2007).

28. Liang, C. H., Tian, Z. F., Tsuruoka, T., Cai, W. P. \& Koshizaki, N. Blue and green luminescence from layered zinc hydroxide/dodecyl sulfate hybrid nanosheets. I. Photochem. Photobiol A: Chemistry 224, 110-115 (2011).

29. Ganeev, R. A., Ryasnyanskiy, A. I. \& Usmanov, T. Optical and nonlinear optical characteristics of the Ge and GaAs nanoparticle suspensions prepared by laser ablation. Opt. Commun. 272, 242-246 (2007).

30. Ostwald, W. Studien über die Bildung und Umwandlung fester Körpe. Z. Phys Chem. 22, 289-330 (1897).

31. Bunday, F. P. \& Kasper, J. S. A new dense form of solid germanium. Science 139 340-342 (1963).

32. Jamieson, J. C. Crystal structures at high pressures of metallic modifications of silicon and germanium. Science 139, 762-764 (1963).

33. Bates, C. H., Dachille, F. \& Roy, R. High-pressure transitions of germanium and a new high-pressure form of germanium. Science 147, 860-862 (1965).
34. Qadri, S. B., Skelton, E. F. \& Webb, A. W. High pressure studies of Ge using synchrotron radiation. J. Appl. Phys. 54, 3609-3611 (1983).

35. Kobliska, R. J. et al. Raman scattering from phonons in polymorphs of $\mathrm{Si}$ and $\mathrm{Ge}$. Phys. Rev. Lett. 29, 725-728 (1972).

36. Chung, S. Y., Kim, Y. M., Kim, J. G. \& Kim,Y. J. Multiphase transformation and Ostwald's rule of stages during crystallizaiton of a metal phosphate. Nature Phys. 5, 68-73 (2009).

37. Washington, A. L. et al. Ostwald's rule of stages and its role in CdSe quantum dot crystallization. J. Am. Chem. Soc. 134, 17046-17052 (2012).

38. Gibson, N. et al. Colloidal stability of modifled nanodiamond particles. Diamond Relat. Mater. 18, 620-626 (2009).

39. Wan, Q. et al. Linear and third-order nonlinear optical absorption of amorphous Ge nanoclusters embedded in $\mathrm{Al}_{2} \mathrm{O}_{3}$ matrix synthesized by electron-beam coevaporation. Appl. Phys. Lett. 82, 3162-3164 (2003).

40. Segura, R. A., Reyes-Gasga, J. \& Cárdenas-Triviño, G. Germanium nanoparticles from solvated atoms: synthesis and characterization. Colloid. Polym. Sci. 283, 854-861 (2005).

41. Wilcoxon, J. P., Provencio, P. P. \& Samara, G. A. Synthesis and optical properties of colloidal germanium nanocrystals. Phys. Rev. B 64, 035417 (2001).

42. Heath, J. R., Shiang, J. J. \& Alivisatos, A. P. Germanium quantum dots: optical properties and synthesis. J. Chem. Phys. 101, 1607-1615 (1994).

43. Lu, X. M., Ziegler, K. J., Ghezelbash, A., Johnston, K. P. \& Korgel, B. A. Synthesis of germanium nanocrystals in high temperature supercritical fluid solvents. Nano Lett. 4, 969-974 (2004).

44. Chou, N. H., Oyler, K. D., Motl, N. E. \& Schaak, R. E. Colloidal synthesis of germanium nanocrystals using room-temperature benchtop chemistry. Chem. Mater. 21, 4105-4107 (2009).

45. Ruddy, D. A., Johnson, J. C., Smith, E. R. \& Neale, N. R. Size and bandgap control in the solution-phase synthesis of near-infrared-emitting germanium nanocrystals. ACS Nano 4, 7459-7466 (2010).

46. Meléndrez, M. F., Cárdenas, G. \& Palencia, M. S. Colloidal germanium nanoparticles and nanotubes synthesized by chemical liquid deposition. Adv. Sci. Lett. 4, 526-535 (2011).

47. Fang, Y. X. \& Al-Abed, S. R. Correlation of 2-chlorobiphenyl dechlorination by $\mathrm{Fe} / \mathrm{Pd}$ with iron corrosion at different pH. Environ. Sci. Technol. 42, 6942-6948 (2008).

48. Kim, Y. H. \& Carraway, E. R. Dechlorination of pentachlorophenol by zero valent iron and modified zero valent irons. Environ. Sci. Technol. 34, 2014-2017 (2000).

49. Farrell, J., Kason, M., Melitas, N. \& Li, T. Investigation of the long-term performance of zero-valent iron for reductive dechlorination of trichloroethylene. Environ. Sci. Technol. 34, 514-521 (2000).

50. Dombek, T., Dolan, E., Schultz, J. \& Klarup, D. Rapid reductive dechlorination of atrazine by zero-valent iron under acidic conditions. Environ. Pollut. 111, 21-27 (2001).

51. Sayles, G. D., You, G. R., Wang, M. X. \& Kupferle, M. J. DDT, DDD and DDE dechlorination by zero-valent iron. Environ. Sci. Technol. 31, 3448-3454 (1997)

52. Li, M. S. et al. Epoxy-polycarbonate blends catalyzed by a tertiary amine. 1 . mechanism of transesterification and cyclization. Macromolecules, 29, 499-506 (1996).

53. Tian, Z. F., Liang, C. H., Liu, J., Zhang, H. M. \& Zhang, L. D. Reactive and photocatalytic degradation of various water contaminants by laser ablationderived $\mathrm{SnO}_{\mathrm{x}}$ nanoparticles in liquid. J. Mater. Chem. 22, 17210-17214 (2011).

54. Weckhuysen, B. M., Wachs, I. E. \& Schoonheydt, R. A. Surface chemistry and spectroscopy of chromium in inorganic oxides. Chem. Rev. 96, 3327-3350 (1996).

\section{Acknowledgments}

We thank the National Key Basic Research Program of China (Grant no. 2013CB934304), the National Science Foundation of China (Grant no. 11174287, 50931002, 11204308) and the Hundred Talent Program of Chinese Academy of Sciences for financial support.

\section{Author contributions}

J.L. performed the experiments, analyzed the data. C.H.L. designed the experiments, analyzed the results and wrote the paper. Z.F.T. helped with synthesis of the materials. S.Y.Z. helped collect and analyze the HRTEM data. G.S.S. analyzed the results and revised the paper. All authors reviewed the manuscript.

\section{Additional information}

Supplementary information accompanies this paper at http://www.nature.com/ scientificreports

Competing financial interests: The authors declare no competing financial interests.

License: This work is licensed under a Creative Commons

Attribution-NonCommercial-NoDerivs 3.0 Unported License. To view a copy of this license, visit http://creativecommons.org/licenses/by-nc-nd/3.0/

How to cite this article: Liu, J., Liang, C.H., Tian, Z.F., Zhang, S.Y. \& Shao, G.S. Spontaneous Growth and Chemical Reduction Ability of Ge Nanoparticles. Sci. Rep. 3, 1741; DOI:10.1038/srep01741 (2013). 